\title{
Coupling Computational and Intracellular Screening and Selection Towards Co-compatible cJun and cFos Antagonists
}

\author{
Alexander Lathbridge ${ }^{1}$, Anna S. Michalowska ${ }^{1,2}$, and Jody M. Mason ${ }^{1, *}$ \\ ${ }^{1}$ Department of Biology \& Biochemistry, University of Bath, Claverton Down, Bath, BA2 7AY, UK \\ 2Present address: AGC Biologics, Vandtaarnsvej 83B, 2860 Soeborg, Copenhagen, Denmark \\ *Corresponding author: Jody M. Mason (E: j.mason@bath.ac.uk; T: +441225386867)
}

Keywords: Coiled coil; Activator Protein-1; transcription factor; PCA, computational design, peptide libraries, protein-protein interactions 


\section{Supporting Information}

Contained within the supporting information are DNA sequencing results and chromatograms, information on the Fos-Library used for the PCA stages, and mass spectrometry data relating to the synthetic peptides.

$\begin{array}{cccccccc} & \text { g0 } & \text { a1 } & \text { b1 } & \text { c1 } & \text { d1 } & \text { e1 } & \text { f1 } \\ \text { P0 } & \text { Q } & \text { L } & \text { D } & \text { T } & \text { L } & \text { Q } & \text { A } \\ \text { P1 } & \text { E } & \text { I } & \text { D } & \text { T } & \text { L } & \text { E } & \text { A } \\ \text { P2 } & \text { E } & \text { I } & \text { D } & \text { T } & \text { L } & \text { E } & \text { A } \\ \text { Options } & \text { EKQ } & \text { IL } & & & & \text { EKQ } & \\ & & & & & & & \\ \text { P0 } & \text { g1 } & \text { a2 } & \text { b2 } & \text { c2 } & \text { d2 } & \text { e2 } & \text { f2 } \\ \text { P1 } & \text { E } & \text { I } & \text { D } & \text { Q } & \text { L } & \text { E } & \text { D } \\ \text { P2 } & \text { E } & \text { L } & \text { D } & \text { Q } & \text { L } & \text { E } & \text { D } \\ \text { Options } & & \text { IL } & & \text { Q } & \text { L } & \text { E } & \text { D }\end{array}$

\begin{tabular}{|c|c|c|c|c|c|c|c|}
\hline & g2 & a3 & b3 & c3 & d3 & e3 & f3 \\
\hline PO & $\mathrm{K}$ & $\mathrm{N}$ & $Y$ & $A$ & $\mathrm{~L}$ & $\mathrm{~K}$ & $\mathrm{~T}$ \\
\hline P1 & $\mathrm{K}$ & $\mathrm{N}$ & $Y$ & A & $\mathrm{L}$ & $Q$ & $\mathrm{~T}$ \\
\hline P2 & $Q$ & $\mathrm{~N}$ & $Y$ & $A$ & L & K & $\mathrm{T}$ \\
\hline \multirow[t]{2}{*}{ Options } & EKQ & IN & & & & EKQ & \\
\hline & g3 & a4 & b4 & c4 & d4 & e4 & f4 \\
\hline PO & $\mathrm{E}$ & $\mathrm{H}$ & $A$ & $\mathrm{~N}$ & L & $\mathrm{E}$ & $\mathrm{K}$ \\
\hline P1 & $E$ & L & $A$ & $\mathrm{~N}$ & $\mathrm{~L}$ & $E$ & $\mathrm{~K}$ \\
\hline P2 & $\mathrm{E}$ & L & A & $\mathrm{N}$ & L & E & $\mathrm{K}$ \\
\hline Options & & ILN(H & & & & & \\
\hline
\end{tabular}

\begin{tabular}{c|cccccc} 
& g4 & a5 & b5 & c5 & d5 & e5 \\
P0 & Q & I & E & K & L & Q \\
P1 & Q & L & E & K & L & Q \\
P2 & E & I & E & K & L & Q \\
Options & EQ & IL & & & & EKQ
\end{tabular}

Figure S1. Overview of PCA passages. The options (red) were randomised at 11 positions and the residue selections for each stage shown in blue. At both $a^{3}$ and $e^{5}$, there were no changes in residue from the first passage (Asn and Gln, respectively). 
Fos-Library. The degenerate DNA sequence was used to define the library used at the PCA stages.

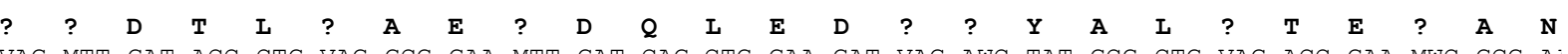
VAG MTT GAT ACC CTG VAG GCG GAA MTT GAT CAG CTG GAA GAT VAG AWC TAT GCG CTG VAG ACC GAA MWC GCG Ai BTC KAA CTA TGG GAC BTC CGC CTT KAA CTA GTC GAC CTT CTA BTC TWG ATA CGC GAC BTC TGG CTT KWG CGC TI $\begin{array}{llllll}\text { EKQ IL } & \text { EKQ } & \text { IL } & \text { EKQ IN } & \text { EKQ }\end{array}$

Figure S2: The degenerate library shown was created from the 34 unique isPCA/isCAN sequences to result in a small high quality and PCA-accessible library of 23,328 sequences, which was expanded further to 31,104 sequences with the addition of His at one core position.
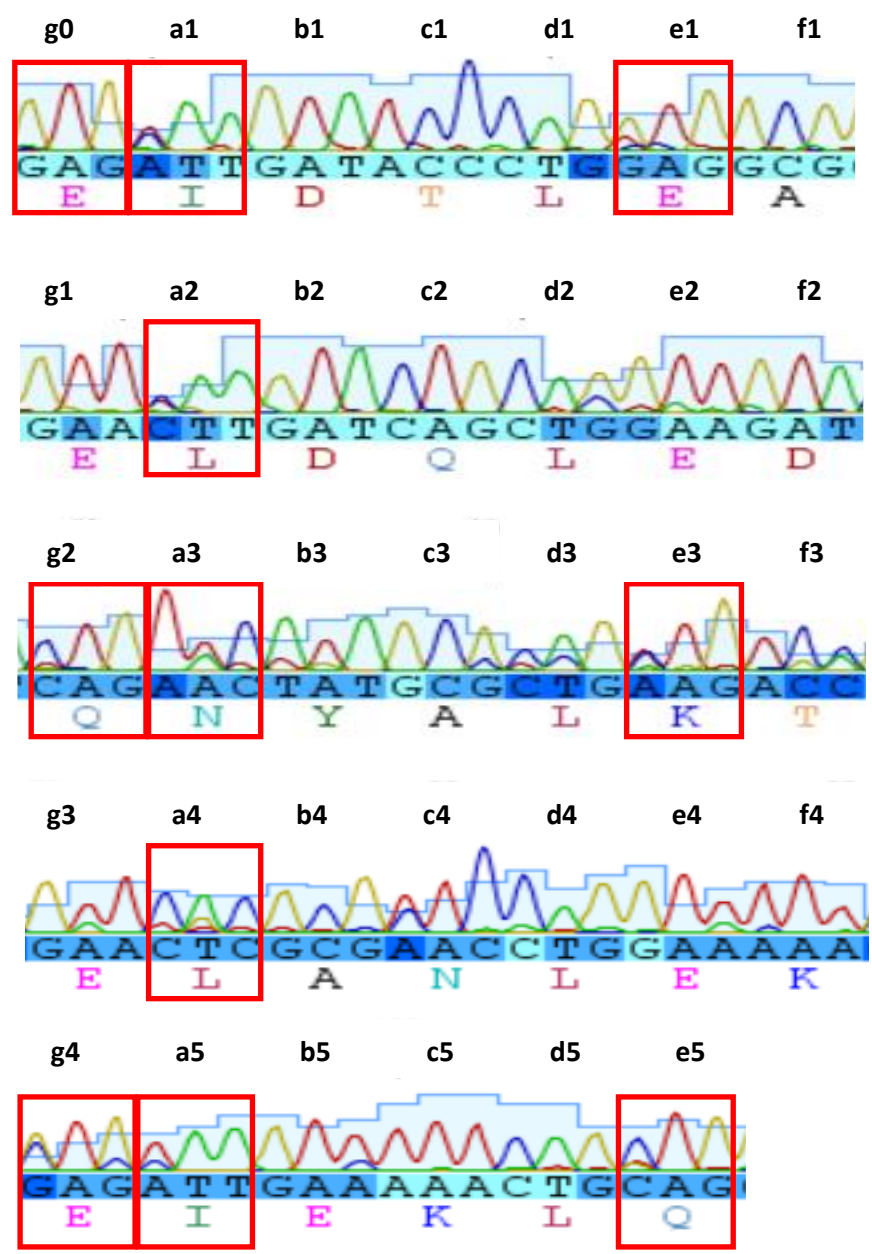

Figure S3. Fos Library Chromatogram. Sequence data from the second (and final) passage (P2) showed the selection results from 11 randomised positions in the sequence (highlighted in red). Glu residues were selected at $g^{0}, e^{1}$, and $g^{4}$ whereas Lys was selected at $e^{3}-$ with $\mathrm{Gln}$ residues on $g^{2}$ and $e^{5}$. lle was chosen at $a^{1}$ and $a^{5}$, Leu at $a^{2}$ and $a^{4}$, and Asn at $a^{3}$. 
(A)

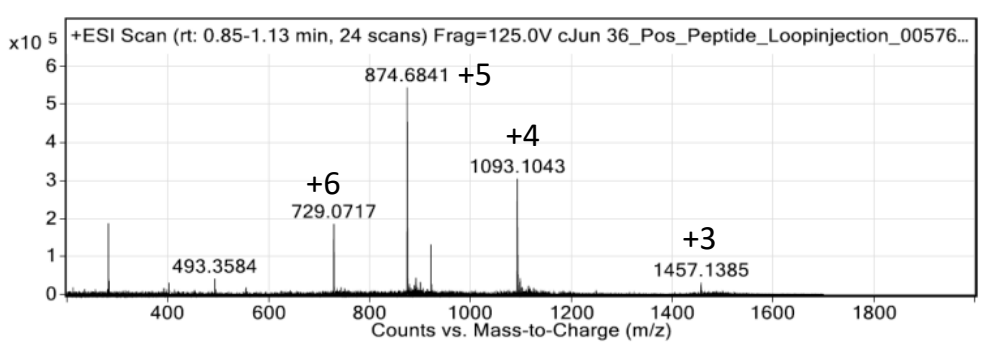

cJun: $\mathrm{C}_{189} \mathrm{H}_{323} \mathrm{~N}_{57} \mathrm{O}_{59} \mathrm{~S}_{1}$

(B)

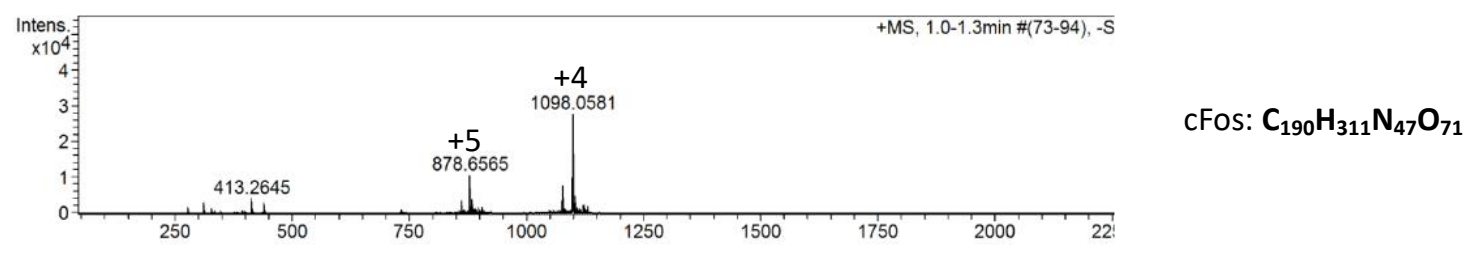

Figure S4. Electrospray Mass Spectrometry data from purified cJun and cFos demonstrating correct $+3-+5 \mathrm{~m} / \mathrm{z}$ of cJun $\mathrm{A}$ and cFos (B)

(A)

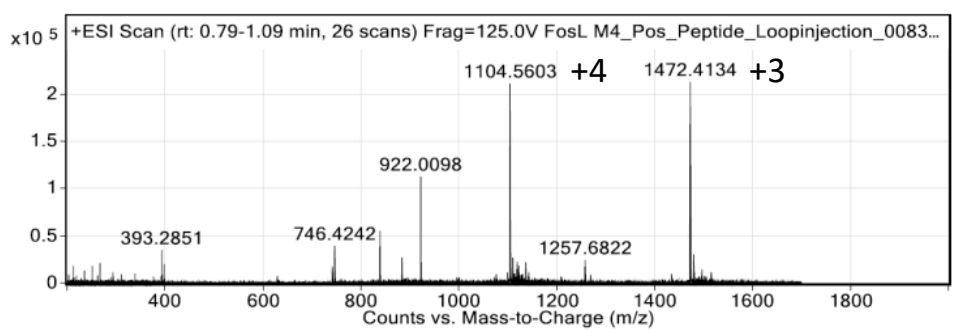

FosU $U_{P C A}: \mathrm{C}_{191} \mathrm{H}_{310} \mathrm{~N}_{48} \mathrm{O}_{71}$

(B)

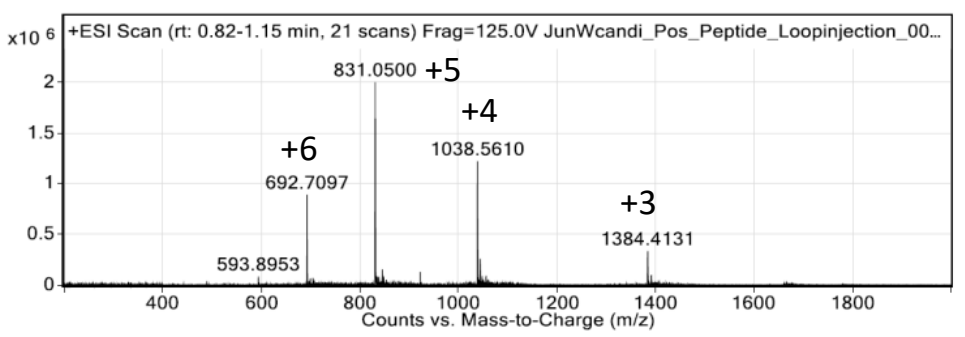

JunW $\mathrm{CANDI}_{\mathrm{C}} \mathrm{C}_{180} \mathrm{H}_{302} \mathrm{~N}_{54} \mathrm{O}_{56} \mathrm{~S}_{1}$

Figure S5. Electrospray Mass Spectrometry data from purified Fos $U_{\mathrm{PCA}}$ and JunW $\mathrm{W}_{\mathrm{CANDI}}$ demonstrating correct $+3-+5 \mathrm{~m} / \mathrm{z}$ of Fos $\mathrm{UPCA}_{\mathrm{PCA}}(\mathrm{A})$ and JunW $\mathrm{W}_{\text {CANDI }}(\mathrm{B})$ 